\title{
Recent Progress on Endogeneity in Choice Modeling
}

By Jordan Louviere (University of Technology, Sydney), ${ }^{1}$ Kenneth Train (University of California, Berkeley), Moshe Ben-Akiva (MIT), Chandra Bhat (University of Texas, Austin), David Brownstone (University of California, Irvine), Trudy Ann Cameron (University of Oregon), Richard T. Carson (University of California, San Diego), J.R. DeShazo (University of California, Los Angeles), Denzil Fiebig (University of New South Wales), William Greene (New York University), David Hensher (University of Sydney), and Donald Waldman (University of Colorado, Boulder)

\begin{abstract}
We describe recent progress in several areas related to endogeneity, including: choice set formation and attention to attributes; interactions among decision-makers; respondents’ strategic behavior in answering stated preference choices; models of multiple discrete/continuous choice; distributions of willingness-to-pay; and methods for handling traditionally endogenous explanatory variables.
\end{abstract}

${ }^{1}$ Corresponding author. Email: deci@bigpond.net.au. Address: Faculty of Business, University of Technology, Sydney, Haymarket Campus, PO Box 123, Broadway NSW 2007, Australia. Voice: 61-2-8586-5600. 
Workshop participants agreed that a working definition of "endogenous" is "all effects that are not exogenous," whereby endogeneity is the same as model misspecification and ignoring it can be expected to lead to biased coefficient estimates and inferences. This broad definition can be linked to the traditional textbook definition whereby endogeneity consists of correlation between the errors and observed variables in the model, since misspecification generally induces nonindependent errors. We take this broad view because the attempts that we made at restriction drew legitimate objections from one or more participants as excluding important and relevant topics. Research progress was reported in several specific areas that relate to endogeneity. In behavioral theory, Cameron and DeShazo (2004) presented an interesting conceptual framework derived from first principles that explains how individuals form consideration/choice sets and focus on subsets of attributes. Hensher and Pucket (2004) discussed several issues in understanding and modeling interactive agency choices resulting from interactions and/or bargaining between two or more agents. Carson, et al. (1999) discussed a theory of incentive compatibility of survey questions for public and private goods. Bhat (2005) discussed a random utility theory-based model for multiple discrete/continuous choice outcomes, including (potentially) choice set formation. We discuss these and other developments in the sections below.

\section{Choice Set Formation and Attribute Information Processing}

Choice modeling analysts typically specify a given set of exogenous options from which all individuals are assumed to choose, and full information is assumed. In reality, however, individuals may decide how to acquire and use information about choice sets, a process that makes the effective choice set endogenous. To correct for this type of endogeneity, one must model the process by which individuals construct and attend to information in choice sets. 
Manski (2004) discusses related issues, and notes the implausibility of many assumptions associated with rational expectations in choice models.

Thus, one should try to avoid biases due to omitting relevant choice options or including irrelevant options (Williams and Ortuzar 1982; Hicks and Strand, 2000). Some researchers have tried to elicit relevant consideration sets directly from choosers (Peters et. el., 1995; Hicks and Strand, 2000), although Horowitz and Louviere (1995) question whether this provides the desired information. Instead, one could formally model the composition of consideration sets as an endogenous part of the overall choice process. This would allow systematic variation in the probability that a given alternative will be considered by an individual as a function of exogenous variables like prices/costs, travel time limits, distances, and restrictions imposed by other agents. This approach has been widely used to estimate revealed-preference choice data models in transportation, geography, marketing, and environmental valuation (Swait and BenAkiva, 1987; Horowitz, 1991; Ben-Akiva and Boccara, 1995; Haab and Hicks, 1997; Dai, 1998; Swait, 2001; and Basar and Bhat, 2004).

Cameron and DeShazo (2004, hereafter CDS), building on work by Gabaix et al. (2003) and DeShazo and Fermo (2004), propose a new approach based on the idea that individuals consider the costs and benefits of processing information in a given choice set. Their ideas can be applied in real choice settings, but are most easily implemented in stated-preference (SP) settings where researchers have designed choice sets that individuals face on given choice occasions. A consumer's problem is to optimally allocate attention, to different options and to their various attributes, subject to constraints on the total amount of cognitive effort that is allocated to each choice occasion. This leads to implicit attention weights associated with each attribute of each choice option, on which observed choices can be conditioned. 
CDS suggest that the cost of attention is determined by a subject's cognitive abilities, opportunity costs of time, and the quantity and structure of choice set information (Dellaert, et al., 1999; Iglesias-Parro et al, 2002); the benefits of attention come from avoided utility loss from incorrect choices. In binary choice contexts CDS show that the marginal benefit of attending to an additional attribute should depend on: a) the absolute value of differences across options in the utility contribution of that attribute (where the utility contribution is the level of an attribute times its marginal utility), and b) how two options differ with respect to the utility to be derived from all attributes other than the attribute in question. Both of these determinants depend on preferences and how they interact with the configuration of the choice set design.

Failure to recognize selective allocation of attention to information in a choice set may bias utility parameter estimates. CDS note that if increased attention costs result in nonproportional changes in attention, there may be significant consequences for welfare estimates. For example, cognitive optimization might induce subjects to reduce attention only to selected non-cost attributes, creating systematic attenuation in their apparent marginal utilities, which would bias the apparent willingness to pay for their attributes downward. Finally, CDS suggest that attention to attributes and choice options can be deliberately influenced by the design of the choice sets used in the SP experiment.

\section{Interactions among decision makers}

The workshop emphasized endogeneity for single-agent choices, but we also considered group decisions by two or more agents. Unlike Adamowicz et al. (this issue), Hensher and Puckett (2004) and Bartels et al. (2005) provide two perspectives on the role and influence of one agent on the decision-making of a second agent. They separate agent interaction processes from outcomes of such processes. The processes can be related to various interactive cases, like 
retailers, suppliers and transporters in freight distribution chains; plumbers giving expert advice to households buying durables (e.g., water heaters); and household members involved in decisions to replace automobiles.

Relationships between agents can take a number of forms, principally bargaining and non-bargaining interactions. Conceptual frameworks to represent how two or more agents interact, and influence the choices made by each, must recognize several endogenous and exogenous interactions. Such interactivity can take many forms empirically, but can be synthesized into three main types, as enumerated by Manski (2000): “1) endogeneous interactions, wherein the propensity of an agent to behave in some way varies with the behavior of the group; 2) contextual interactions, wherein the propensity of an agent to behave in some way varies with exogenous characteristics of the group members; 3) correlated effects, wherein agents in the same group tend to behave similarly because they have similar individual characteristics or face similar institutional environments.”

These notions pose several challenges for econometric representations that can separately identify these effects. One way forward suggested by Manski (2004) is to use experimental and SP data. An example is provided by Bartels, et al. (2004), who use SP methods to model an endogenous interaction while avoiding econometric endogeneity problems that must be addressed in revealed preference (RP) settings.

It is problematic to try to infer the nature of interaction processes from outcome observations alone in either SP and RP contexts. It is critical to identify process strategies that agents bring to the interactive games used to define the operating rules. Interactive agency 'games' are designed to yield agreement outcomes of a cooperative or non-cooperative form that may be suboptimal from any agent's individual utility-maximization position. We term this an 
agent's information processing strategy (IPS). The choice of an IPS results from a utility maximization exercise for each agent because when choosing among IPSs, agents should choose a strategy that maximizes their utility associated with the entire interactive decision-making process at hand (Hensher and Puckett 2004).

To complete the framework, one requires an optimization function whereby an agent's strategy, in a given round of interactions, is specified as the choice of the optimal act, conditional on the agent's beliefs and IPS. This function is the product of a utility function and a second function that represents the shadow value (to the player's future relationship) of a particular act. That is, the second function measures the benefit/disbenefit that agents think particular currentround negotiation acts will deliver to future agent interactions. For example, an agent may think that accepting another agent's offer will have lasting benefits to the relationship, or an agent may think that conceding to the preferences of another agent will set a precedent enabling the other agent to demand more in the future. The utility function is specified to allow for the possibility that potential future losses carry more weight than potential future gains (Kahneman and Tversky, 1979; Gilboa et al. 2002) and potential losses include the possibility of termination of relationships and a lack of cooperation by other agents in future negotiations.

The workshop identified the need to establish sources of influence on the choice process that conditions choice outcomes, and specifying model systems in such a way that one can minimize the endogeneity in relationships between the process and the outcome.

\section{Strategic Behavior}

The typical analysis of choice data, whether from observed behavior or survey responses, assumes that agents truthfully reveal their preferences. This assumption implies a form of procedural invariance, namely that the choice set format by which preference information is 
obtained does not matter. This might be true in particular instances, but economic theory suggests that it is not generally true. The theoretical framework of incentives and strategic behavior predicts that revealed preference information should differ due to incentives provided by particular response formats. That is, unless formats in which information is collected are "incentive compatible," it may not be in the strategic interest of agents to truthfully reveal information about preferences. Many types of SP questions that have been used in practice are not incentive compatible, and in some cases feasible incentive compatible questions do not exist even though an SP question can still yield valuable information that bounds the quantity of interest. While strategic behavior is most often discussed in a survey context, it also pervades much of observed choice data particularly in the realm of voting or when a static rather than dynamic perspective is adopted.

A few examples from the voting literature illustrate the incentive-compatibility problem. Consider a case in which there are two ballot propositions dealing with the same issue. If both propositions pass, the one with the highest number of votes will be the controlling law. If an agent prefers both propositions to the status quo but has a clear preference for one proposition, how should the agent vote? This depends on the voter's beliefs about what other voters are likely to do. Political scientists show various types of behavior that might be deemed strategic or involve information processing shortcuts to be rampant in this case (Lupia and McCubbins, 1998). Indeed, a popular explanation for the dynamics of U.S. presidential primaries is that there is a front runner and one main challenger (from a pack of challengers). Voters not favoring the front runner tend to flock to the leading challenger whom the media identifies as having the best chance to unseat the front runner. These situations are formally equivalent to multi-candidate 
elections with a single winner, and in such situations it is sometimes in a voter's best interest to vote against their most preferred choice.

Now consider an SP experiment in which subjects are offered a series of choices where the other attributes of different available products stay fixed but prices vary. Subjects are likely to realize that such surveys are designed to determine the price sensitivity of product purchases. A simple utility-enhancing response strategy is to state an intention not to purchase the good if the offered price appears to be high relative to the current real-market offer price. Such response patterns yield the result that subjects seem more price sensitive in surveys than in real markets.

A third example is an SP experiment where subjects are offered a new transportation option in addition to one or more existing options. Subjects are asked which option they would choose as travel times and costs vary. Now it is optimal for subjects to choose the new transport option if there is any positive probability that they may want to use the new option in the future. This behavior will result in new product demand forecasts that are too optimistic.

It is interesting to note that the stylized facts in the second and third examples appear to conflict unless one realizes that, from the perspective of survey subjects, consumers' responses to surveys about existing products mainly influence price, while consumers' responses to surveys about new products mainly influence whether they are introduced into the market. Rational consumers realize this and shift their response strategies accordingly.

Strategic behavior differs from the random behavior that survey researchers often fear and that psychologists contend underlies many consumer choices. Tests that compare RP and SP data (e.g., Carson, et al., 1996; Swait and Louviere, 1993) or those that compare data from different elicitation formats (Cameron, et al., 2002) are more tests against random behavior than tests against strategic non-truthful preference revelations. The later behavior can manifests itself 
in the often observed result that while there are differences in "scale parameters" between two sources of data, the ratio of parameters on non-price attributes is essentially the same in the two data sources. Of course, there are many other reasons why scales in two different situations might vary, but this case is one to be concerned about.

Careful attention to potential incentives for non-truthful preference revelation can help researchers devise improved data collection procedures. This is particularly true for stated preference surveys, where there is considerable flexibility in the nature of the questions posed. More generally, an understanding of strategic incentives can help researchers interpret the results obtained from different choice experiments by helping to predict the ways in which results from two data collection efforts can be expected to differ a priori.

According to Carson, et al. (1999), a relatively small number of factors interact to define incentive structures, such as response formats (e.g., binary, multinomial, open-ended), whether goods are pure public goods, whether goods are provided with a coercive payment mechanism irrespective of actual use (e.g., a tax on a utility bill or a purchase price) or whether they are made available independent of an actual payment (e.g., the transport example above or goods provided by voluntary charitable contributions), and whether uncertainty about goods is resolved ex ante or ex post. Examination of possible incentive structures often reveals interesting biasversus-variance tradeoffs. Variations in these different factors can be important sources of endogeneity that influence the nature of the choice data collected.

\section{Models of multiple discrete/continuous choice}

Traditionally, discrete and discrete-continuous choice models have dealt with cases in which only one option is chosen from a set of mutually exclusive options. However, many decisions are characterized by a simultaneous choice of multiple options. As in traditional simultaneous 
equations, modeling the dependence of one choice on other choices without reference to the endogeneity of the other choices induces inconsistency. Little progress has been made in modeling "multiple discreteness." Models for these processes assume that options are perfect substitutes. Yet, in many cases there is simultaneous demand for multiple options, corresponding to cases where options are imperfect substitutes for one another.

Several recent marketing papers deal with multiple-discreteness, such as Hendel (1999) and Dube (2004), who consider purchases of multiple varieties within particular product categories resulting from a stream of expected future consumption choices between successive shopping occasions (see also Walsh, 1995). Because tastes vary across individual consumption occasions between current and upcoming purchases, consumers are observed to purchase a variety of goods on the current shopping occasion. These studies assume a linear utility function at each individual consumption occasion, with utility parameters varying over consumption occasions, and assume Poisson distributions for numbers of consumption occasions and normal distributions for taste variation to complete the specification of the model.

Such "vertical" variety-seeking models may be suitable for frequently purchased packaged goods like carbonated drinks, cereals, and cookies. However, for many other cases like allocating time to different types of discretionary activities, choice processes may be better characterized as "horizontal” variety-seeking, where consumers select an assortment of options due to diminishing marginal returns for each alternative. Kim et al. (2002) propose utility structures for "horizontal” variety-seeking with non-linear utility functions that capture satiation behavior, but due to econometric and computational complexity, their model is not practical for most realistic applications. 
Recently, Bhat (2005) has derived a model of multiple discreteness that assumes (like Kim et al., 2002) a translated non-linear, but additive, utility specification. The translated nonlinear form allows for multiple discreteness and diminishing marginal returns (i.e., satiation) as the consumption of any particular option increases. This specification is termed a Multiple Discrete-Continuous Extreme Value (MDCEV) model, because a multiplicative logarithmicextreme value error term is assumed in the utility function. This leads to a surprisingly simple closed form expression for the discrete-continuous probability of consuming zero quantities of certain options and consuming given levels of the remaining options. The MDCEV model also collapses to the multinomial logit (MNL) choice model in the case of single discreteness, and is an extension of single discrete-continuous models (MDCEV models are MNL model-equivalents for multiple discrete-continuous choices). It also is relatively easy to specify heteroscedasticity and/or correlation in unobserved variables affecting the demand for different options in the MDCEV framework. This extension results in a multiple discrete-continuous equivalent of mixed multinomial logit (MMNL) (see Bhat, 2003 or Train, 2003 for discussions of the MMNL model). The MDCEV is potentially useful in numerous settings, such as, for example, when examining the amount of time that travelers spent at each destination, when the time spent at one destination can enhance or diminish the experience at another destination.

\section{Distributions of coefficients and distributions of willingness to pay}

A consumer's willingness to pay for a marginal improvement in an attribute of an option, conditional on the consumer choosing that option is the ratio of the attribute's coefficient to the price coefficient. Choice models usually are parameterized in term of utility coefficients, with willingness to pay (WTP) calculated from the estimated coefficients. Cameron and James (1987) and Cameron (1988) alternatively parameterize choice models in terms of WTP; that is, each 
attribute's coefficient in the conventionally parameterized random utility model is expressed instead as the product of WTP for the attribute times the negative of the price coefficient, allowing WTP to be estimated directly. For fixed-coefficient models, these two parameterizations differ mainly in terms of the ease of estimation of standard errors for WTP. These standard errors are easier to calculate and require fewer approximations if model parameters directly estimate the marginal WTP.

For random coefficient models the issue of which parameterization to use is more complex and potentially more important, as noted by Sonnier et al., (2003) and Train and Weeks (2004). Models parameterized in coefficient distributions are called "models in preference space," and models parameterized in WTP distributions are "models in WTP space.” Mutually compatible distributions for coefficients and WTP's can be specified either way, but differ in their convenience for assigning parameter distributions and imposing constraints on these distributions.

Sonnier, et al. (2003) and Train and Weeks (2004) compare models using normal and lognormal distributions in preference space with those using normal and lognormal distributions in WTP space. Both studies found that models in preference space fit the within-sample data better than models in WTP space using different data sets; both studies also found that distributions of WTP derived from estimated models in preference space have unreasonably large variances. In particular, models in preference space imply that large proportions of choosers will pay unreasonably large sums to obtain/avoid extra units of non-price attributes. Models in WTP space exhibited smaller variances for WTP, implying smaller proportions of very large WTP values. These results suggest that better fitting models have less realistic distributions of WTP, in turn suggesting a need to specify distributions that fit data better in 
WTP space or yield more reasonable WTP distributions derived from models specified in preference space.

Many choice models with random coefficients specify price coefficients as fixed (e.g., Goett et al., 2000; Hensher et al., 2004.), which is a useful constraint as it implies that the distribution of marginal WTP takes the same form as the distributions of the corresponding attribute coefficient. This constraint also tends to avoid unreasonably large variances in WTP for models in preference space because the distribution of WTP is not a convolution of two distributions. However, a fixed price coefficient (or any fixed coefficient) implies that scale parameters (i.e., error standard deviations) are the same for all observations. Louviere (2003) notes that scale parameters vary in many settings like SP tasks where scale represents pure randomness in respondents' choices instead of variation in factors known to decision-makers but not observed by researchers. If one needs random scale parameters to appropriately represent choice processes, issues identified by Sonnier, at al. (2003) and Train and Weeks (2004) become critical, motivating future research on this unresolved problem.

\section{Endogenous explanatory variables}

As stated in the introduction, the term endogeneity usually refers to situations where observed explanatory variables are correlated with error terms, so that standard estimation procedures that rely on independent errors cannot be used directly. The classical form of endogeneity arises in random utility models if variables that enter systematic utility components are correlated with random utility components, such as: 1) Some relevant product attributes might be omitted in product characterizations; for example, car prices depend on both observed and unobserved attributes because attributes affect manufacturer costs and/or price equilibration processes. 2) In work mode choices, each person's travel cost and transit time can depend on unobserved 
preferences because people who like transit also tend to choose housing close to transit. 3) In SP tasks, subjects may infer differences in omitted attributes from differences in the levels of included attributes. For example, if the price of an option is higher than a subject thinks it should be based on just the included non-price attributes, a subject may infer that there must be some omitted attributes that explain the unexpectedly high price.

An attractive and widely used approach for dealing with endogeneity in a market setting is the BLP approach (Berry 1994 and Berry et al., 1999). The BLP strategy moves the endogeneity out of nonlinear choice models into linear regressions, allowing standard instrumental variables methods to be used. BLP procedures are applicable when endogeneity arises over groups of decision-makers, e.g., when price is set separately in geographical markets where each has many consumers, and price and unobserved attributes vary only across these markets but are the same for all customers in the same market. BLP is particularly useful because the distribution of errors around their (zero) conditional means need not be known or estimated.

Villas-Boas and Winer (1999) and Blundell and Powell (2001) proposed a control function approach. The endogenous variable is regressed against exogenous instruments, and the residual from this regression (or a function of the residual) is entered as an additional explanatory variable in utility, which is called the control function. Including the new variable induces a new error component, whose distribution must be known or empirically inferred to derive choice probabilities.

Matzkin (2004) proposes a "dual" approach to handling endogeneity in linear and nonlinear models. In standard instrumental variables (IV) approaches (e.g., IV in linear models or control functions), researchers must find exogenous variables not already in the model that are correlated with endogenous explanatory variables. Instead, Matzkin proposes finding variables 
that also are endogenous, but related to the original endogenous variables only through exogenous perturbations. Under certain conditions, she shows that the original error can be expressed as a function of the new endogenous variable and a new error that is independent of all explanatory variables. In choice models, the new variable is included in utility with the original endogenous variable. The distribution of the new error must be known or discovered empirically as in the control function approach, but this approach can be combined with BLP to move the endogeneity out of choice models into linear models, where the distribution of the new error need not be specified. Train and Winston (2004) estimate a model that can be interpreted as being an application of her approach (see their footnote 16 for an interpretation).

Lewbel (2004) proposes using "very exogenous" variables to handle endogenous explanatory variables in binary choice models. A variable is "very exogenous" if the error in a linear regression of that variable against all other explanatory variables in the model is uncorrelated with all explanatory variables. Lewbel shows that 0-1 dependent variables can be transformed with a very exogenous variable such that the transformed dependent variable is linear in other explanatory variables. The model becomes a linear regression wherein the transformed dependent variable is regressed against the original explanatory variables, allowing standard IV methods to be applied.

There are also situations where endogeneity is caused by measurement error, and if auxiliary data are available to identify the measurement error then multiple imputations techniques are a simple approach to obtaining consistent inference (see Brownstone et. al. 2001). This situation frequently occurs in transportation mode choice models where travel times and costs are assigned from network flow models. 
Endogeneity of the various different forms characterized in this paper seems likely to be present in many types of choice situations. Workshop participants agreed that many of these forms of endogeneity have the potential to distort inferences about preferences and therefore to distort the policy or marketing advice that is derived from choice data.

\section{References:}

Bartels, R., Fiebig, D., and A. van Soest. (2004). "Consumers and Experts: An Econometric Analysis of the Demand for Water Heaters,” forthcoming, Empirical Economics.

Basar, G., and C. Bhat. (2004). "A Parameterized Consideration Set Model for Airport Choice," Transportation Research 38B, 889-904.

Ben-Akiva, M., and B. Boccara. (1995). "Discrete Choice Models with Latent Choice Sets,” International Journal of Research in Marketing 12, 9-24.

Berry, S. (1994). "Estimating Discrete Choice Models with Product Differentiation," RAND Journal of Economics 25, 242-262.

Berry, S., J. Levinsohn, and A. Pakes. (1995). "Automobile Prices in Market Equilibrium,” Econometrica 63, 841-889.

Bhat, C.. (2003). "Simulation Estimation of Mixed Discrete Choice Models using Randomized and Scrambled Halton Sequences,” Transportation Research 37B, 837-855.

Bhat, C. (2005). “A Multiple Discrete-Continuous Extreme Value Model: Formulation and Application to Discretionary Time-Use Decisions,” Transportation Research 39B, 679-707.

Blundell, R., and J. Powell. (2001). "Endogeneity in Semiparametric Binary Response Models,” working paper, Economics, University College London. 
Brownstone, D., T. Golob, and C. Kazimi. (2001). "Modeling Non-Ignorable Attrition and Measurement Error in Panel Surveys," Ch. 25 in Survey Nonresponse, R.Groves, D. Dillman, J. Eltinge and R. Little, eds., New York: Wiley, 373-388.

Cameron, T. (1988). “A New Paradigm for Valuing Mon-Market Goods using Referendum Data,” Journal of Environmental Economics and Management 15, 355-379.

Cameron, T., and J. DeShazo. (2004). “Cognitive Resource Constraints, Task Complexity and Attention to Choice-Set Attributes and Alternatives in a Utility-Theoretic Discrete Choice Model," presented at this symposium.

Cameron, T., and M. James. (1987). "Efficient Estimation Methods for Closed-Form Contingent Valuation Survey Data," Review of Economics and Statistics 69, 269-276.

Cameron, T., G. Poe, R. Either, and W. Schulze. (2002). “Alternative Non-Market Elicitation Methods: Are the Underlying Preferences the Same,” Journal of Environmental Economics and Management 44(3), 391-425.

Carson, R., N. Flores, K. Martin and J. Wright. (1996). "Contingent Valuation and Revealed Preference Methodologies," Land Economics 72(1), 80-99.

Carson, R., T. Groves, J. List, and M. Machina. (2004). "Probabilistic Influence and Supplemental Benefits: A Field Test of the Two Key Assumptions Underlying Stated Preferences,” paper presented at NBER Public Economics Workshop, Palo Alto.

Carson, R., T. Groves and M. Machina. (1999). "Incentive and Informational Properties of Preferences Questions,” Plenary Address, European Association of Environmental and Resource Economists, Oslo Norway.

Dai, J. (1998). "Calibration and Test of a Discrete Choice Model with Endogenous Choice Sets,” Geographical Analysis 30, 95-118. 
Dellaert, B., J. Brazell, and J. Louviere. (1999). "The Effect of Attribute Variation on Consumer Choice Consistency,” Marketing Letters 10, 139-147.

DePalma, A., G.M. Myers, and Y.Y. Papageorgiou. (1994). "Rational Choice under an Imperfect Ability to Choose,” American Economic Review 84, 419-440.

DeShazo, J.R. and G. Fermo. (2004). "Implications of Rationally-Adaptive Pre-choice Behavior for the Design and Estimation of Choice Models.” Working paper, UCLA.

DeShazo, J.R. and G. Fermo. (2002). "Designing Choice Sets for Stated Preference Methods,” Journal of Environmental Economics and Management 43(3) 360-385.

Dube, J.P. (2004). "Multiple Discreteness and Product Differentiation: Demand for Carbonated Soft Drinks,” Marketing Science 23(1), 66-81.

Gabaix, X., D. Laibson, G. Moloche, and S. Weinberg. (2003). "The Allocation of Attention: Theory and Evidence,” discussion paper, MIT.

Gilboa, I., D. Schmeidler, and P. Wakker. (2002). "Utility in Case-Based Decision Theory, Journal of Economic Theory, 105, 483-502.

Goett, A., K. Hudson, and K. Train. (2000). “Consumers’ Choice among Retail Energy Suppliers: The Willingness-to-Pay for Service Attributes,” The Energy Journal, 21, 1-28.

Haab, T., and R. Hicks. (1997). “Accounting for Choice Set Endogeneity in Random Utility Models of Recreation Demand,” Journal of Environmental Economics and Management 34, 127-147.

Hendel, I. (1999). "Estimating Multiple-Discrete Choice Models: An Application to Computerization Returns,” Review of Economic Studies 66, 423-446.

Hensher, D., and S. Puckett. (2004). "Agent Interaction and Choice Revelation in the Establishment of Freight Distribution Contracts in Supply Chains," presented at this symposium. 
Hensher, D., N. Shore, and K. Train. (2004). “Households’ Willingness to Pay for Water Service Attributes,"forthcoming, Environmental and Resource Economics.

Hicks, R., and I. Strand. (2000). “The Extent of Information: Its Relevance for Random Utility Models,” Land Economics 76, 374-385.

Horowitz, J. (1991). “Modeling the Choice of Choice Set in Discrete-Choice RandomUtility Models, Environment and Planning A 23, 1237-46.

Horowitz, J., and J. Louviere. (1995) "What is the Role of Consideration Sets in Choice Modeling?,” International Journal of Research in Marketing 12, 39-54.

Iglesias-Parro, S., E. De La Fuente, and A. Ortega. (2002) "The Effect of Context Variables on Cognitive Effort in Multiattribute Binary Choice,” Theory and Decision 52, 101125.

Kahneman, D., and A. Tversky. (1979). "Prospect Theory: An Analysis of Decisions under Risk,” Econometrica 47(2), 263-91.

Kim, J., G. Allenby, and P. Rossi. (2002). “Modeling Consumer Demand for Variety,” Marketing Science 21, 229-250.

Lewbel, A. (2004). "Simple Estimators for Hard Problems: Endogeneity and Dependence in Binary Choice Related Models,” presented at this symposium.

Louviere, J. (2003), "Random Utility Theory-Based Stated Preference Elicitation Methods,” working paper, Faculty of Business, University of Technology, Sydney.

Lupia, A. and M. McCubbins. (1998). The Democratic Dilemma: Can Citizens Learn What They Need to Know. New York: Cambridge University Press.

Manski, C. (2000), “Economic Analysis of Social Interactions,” Journal of Economic Perspectives 14(3), 115-135. 
Manski, C. (2004) “Measuring Expectations,” Econometrica 72(5), 1329-1376.

Matzkin, R. (2004). “Unobservable Instruments,"working paper, Economics, Northwestern University.

Peters, T., W. Adamowicz, and P. Boxall. (1995). "The Influence of Choice Set Considerations in Modelling the Benefits of Improved Water Quality,” Water Resources Research 613, 1781-7

Sonnier, G. A. Ainslee, and T. Otter. (2003). "The Influence of Brand Image and Product Style on Consumer Brand Valuations,” working paper, UCLA.

Swait, J. (2001). “Choice Set Generation within the Generalized Extreme Value Family of Discrete Choice Models,” Transportation Research Part B 35, 643-666.

Swait, J., and M. Ben-Akiva. (1987). "Incorporating Random Constraints in Discrete Models of Choice Set Generation,” Transportation Research Part B 21, 91-102.

Swait, J., and J. Louviere. (1993). "The Role of the Scale Parameter in the Estimation and Use of Multinomial Logit Models." Journal of Marketing Research 30(3), 305-314.

Train, K. (2003). Discrete Choice Methods with Simulation. Cambridge University Press. Train, K., and M. Weeks. (2004). “Discrete Choice Models in Preference Space and Willingnessto-Pay Space,” working paper, Economics, University of Cambridge.

Train, K., and C. Winston. (2004). "Vehicle Choice Behavior and the Declining Market Share of U.S. Automakers,” working paper, Economics, University of California, Berkeley.

Villas-Boas, J., and R. Winer. (1999). "Endogeneity in Brand Choice Models," Management Science, 45, 1324-1338.

Walsh, J. (1995). "Flexibility in Consumer Purchasing for Uncertain Future Tastes," Marketing Science, 14, 148-165. 
Williams, H., and J. Ortuzar. (1982). "Behavioral Theories of Dispersion and the Misspecification of Travel Demand Models,” Transportation Research Part B 16, 167-219. 